\title{
« Rejoindre les civilisations », Victor Brauner et sa collection d'arts extra-européens
}

Victor Brauner (1903-1966) and his collection of extra-European art, a plural relationship

\section{Noémie Fillon}

\section{(2) OpenEdition}

\section{Journals}

Édition électronique

URL : http://journals.openedition.org/cel/4373

DOI : $10.4000 /$ cel. 4373

ISSN : 2262-208X

Éditeur

École du Louvre

Référence électronique

Noémie Fillon, « "Rejoindre les civilisations », Victor Brauner et sa collection d'arts extra-européens », Les Cahiers de l'École du Louvre [En ligne], 14 | 2019, mis en ligne le 02 décembre 2019, consulté le 05 décembre 2019. URL : http://journals.openedition.org/cel/4373 ; DOI : 10.4000/cel.4373

Ce document a été généré automatiquement le 5 décembre 2019

\section{cc) (†)}

Les Cahiers de l'École du Louvre sont mis à disposition selon les termes de la licence Creative Commons Attribution - Pas d'Utilisation Commerciale - Pas de Modification 4.0 International. 


\title{
« Rejoindre les civilisations », Victor Brauner et sa collection d'arts extra-européens
}

\author{
Victor Brauner (1903-1966) and his collection of extra-European art, a plural \\ relationship
}

Noémie Fillon

\section{NOTE DE L'AUTEUR}

Cet article est issu du mémoire de recherche de Master 2 de l'auteure, intitulé Victor Brauner (1903-1966), étude de l'œuvre à l'aune de sa collection d'arts extra-européens, réalisés sous la direction de Camille Morando à l'École du Louvre en 2018. L'auteure tient à remercier Camille Morando et Magali Mélandri pour leurs remarques et suggestions lors de la préparation de cet article.

1 Accroché au-dessus de la cheminée, un masque karanga ${ }^{1}$, exécuté par les Mossi du Burkina Faso, surplombe un crochet de suspension sculpté par les Iatmul de la vallée du Moyen Sépik ${ }^{2}$ et un fragment de sculpture à visage anthropomorphe du Vanuatu ${ }^{3}$. Entre ces deux pièces se trouve une peinture sur tissu de l'artiste Victor Brauner ${ }^{4}$ (fig. 1). Cette photographie, prise dans la maison de ce dernier, à Varengeville-sur-Mer, en Normandie, révèle la mise en scène de sa collection d'arts extra-européens. Brauner juxtapose les aires géographiques et mêle ses propres œuvres à sa collection, favorisant des associations visuelles à même de stimuler sa créativité.

2 Entre le milieu des années 1950 et le début des années 1960, Brauner constitue la majorité de sa collection, principalement composée d'œuvres d'Afrique de l'Ouest et de Papouasie-Nouvelle-Guinée. À cette époque, les collectes intensives et l'instabilité politique liée aux mouvements de décolonisation qui bouleversent le continent africain provoquent une forte affluence des pièces sur le marché de l'art occidental. C'est 
également un moment où «les arts archaïques et primitifs sont à la mode ${ }^{5}$ ». Depuis leur « découverte » au début du siècle par les artistes fauves et cubistes, les arts extraeuropéens sont entrés dans le discours sur l'art moderne ${ }^{6}$. Dès les années 1920, Brauner évolue dans un environnement sensible aux arts africains et océaniens en participant à la scène artistique avant-gardiste de Bucarest ${ }^{7}$, notamment influencée par la pensée du dadaïste Marcel Janco ${ }^{8}$. Après avoir quitté sa Roumanie natale en 1930, il rejoint le mouvement surréaliste parisien en $1933^{\circ}$ et partage l'intérêt de ses membres pour les arts d'Afrique subsaharienne, d'Océanie et d'Amérique. Cet attrait des artistes occidentaux, exprimé à travers leurs œuvres et leurs discours, usuellement nommé " primitivisme $»^{10}$, est complexe à définir, de par les multiples valeurs que cette notion a revêtu à différents moments de l'histoire ${ }^{11}$. Elle témoigne d'un rapport à l'Autre empreint d'une vision coloniale en présupposant la croyance en l'existence d'un art dit "primitif», capable d'offrir une alternative au rationalisme et à l'intellectualisme occidental ${ }^{12}$. L'histoire du paradigme primitiviste de la première moitié $\mathrm{du} \mathrm{xx}^{\mathrm{e}}$ siècle en France a fait l'objet de multiples études ${ }^{13}$, contrairement aux années suivant la Seconde Guerre mondiale ${ }^{14}$. Pourtant, l'intérêt des artistes pour les arts extra-européens persiste et s'inscrit dans un contexte historique, culturel et politique, bien différent. Si de nombreux auteurs ont souligné l'existence d'affinités formelles entre certaines œuvres de Brauner et les arts océaniens ${ }^{15}$, son activité de collectionneur est peu connue ${ }^{16}$.

Cet article se propose de mettre en lumière la constitution de la collection Brauner et les différents types de regards, à la fois distincts et complémentaires, que l'artiste a portés sur les œuvres, afin de rendre compte de la nature plurielle de ses rapports aux arts extra-européens. 
Fig. 1

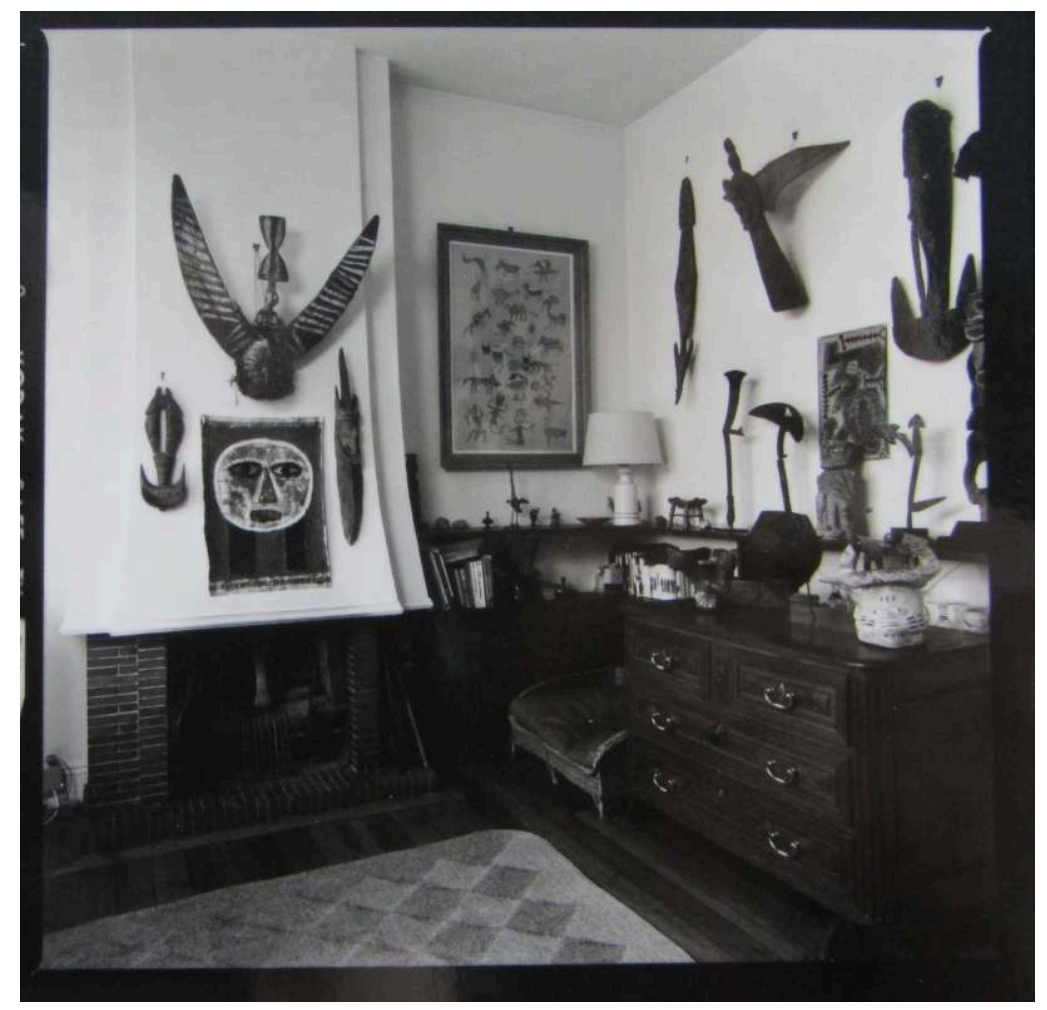

Luc Joubert, L'atelier de Victor Brauner à Varengeville, [c. 1966], Fonds Brauner, BRAU 127, dossier La Normandie, planche contact n 9.107, Paris, Bibliothèque Kandinsky.

(C) Noémie Fillon

\section{La constitution de la collection : le réseau parisien et la galerie Lemaire à Amsterdam}

3 Aujourd'hui, la majeure partie de la collection de Brauner est conservée au musée d'art moderne et contemporain de Saint-Étienne Métropole, léguée par son épouse, Jacqueline Brauner, en 1987. Ce legs se compose de soixante et une œuvres : trente-cinq pièces provenant essentiellement d'Afrique de l'Ouest et des anciennes colonies françaises (Côte d'Ivoire, Cameroun, Mali, Burkina Faso), vingt-trois d'Océanie, majoritairement de Papouasie-Nouvelle-Guinée, deux d'Amérique du Sud (un masque en écorce d'Amazonie et un masque en pierre du Mexique), ainsi qu'une peinture sur écorce d'Australie et une peinture sur papier d'Inde.

4 De nombreuses zones d'ombre entourent la constitution de cette collection. Dans les années 1960, Brauner est proche de plusieurs artistes-collectionneurs surréalistes. Citons André Breton et la poétesse et collectionneuse Joyce Mansour ${ }^{17}$. Leur a-t-il acheté ou échangé des pièces? Sans pouvoir le prouver, il est raisonnable de penser que ces personnalités aient pu participer au développement de la collection. Trois marchands parisiens avec lesquels Brauner est en contact sont plus particulièrement susceptibles de lui avoir vendu ou échangé des pièces : Pierre Loeb, René Rasmussen, et Jean Roudillon. C'est dans la galerie de Pierre Loeb, marchand des surréalistes et collectionneur, que se tient la première exposition personnelle de Brauner, en décembre 1934. Il y exposera à nouveau en mars et avril 1946. Après la guerre, Loeb 
reprend ses activités et présente des œuvres extra-européennes, majoritairement originaires d'Océanie. Y a-t-il eu des transactions d'objets entre Brauner et Loeb ? À ce jour, aucun document ne permet de l'affirmer ${ }^{18}$. Brauner est également proche de René Rasmussen. Reconnu comme l'un des plus importants collectionneurs d'arts extraeuropéens après la Seconde Guerre mondiale, sa galerie est fréquentée par de nombreux artistes surréalistes et par Picasso. Deux tableaux de Brauner portant une dédicace adressée au marchand et à sa femme témoignent de leur amitié19. En 1956, Rasmussen participe à la découverte de l'art dogon en finançant, avec Loeb, la mission de collecte de François Di Dio au Mali. L'expédition dure trois mois et rapporte en France une centaine d'objets. Les cinq statuettes et les trois masques dogon que possède Brauner pourraient provenir de cette expédition. Jean Roudillon est le troisième marchand pour lequel des contacts avec Brauner sont attestés. Il ouvre une première boutique en 1946, puis s'associe à Olivier Le Corneur en 1953 pour proposer des œuvres en provenance d'Afrique subsaharienne et d'Océanie. Lors d'un entretien, il a confié avoir vendu une ou plusieurs pièces à Brauner ${ }^{20}$. Ce témoignage imprécis révèle la difficulté à retracer les acquisitions. Les objets, rarement répertoriés et photographiés, circulaient de mains en mains lors d'échanges ou de ventes à l'amiable.

5 Les seuls achats qui peuvent être documentés avec certitude sont ceux qu'effectue Brauner auprès du marchand Matthias Louis Lemaire à Amsterdam, au cours de l'année 1962. Sa galerie, ouverte en 1933, est l'une des premières de la ville à vendre des œuvres d'Afrique, d'Indonésie et de Papouasie-Nouvelle-Guinée. Lemaire travaille avec des confrères allemands, tels qu'Arthur Speyer et les frères Umlauff, et en Angleterre avec le marchand W. D. Webster. Il est notamment connu pour avoir vendu la collection d'art Sépik du Docteur Lautenbach, ensemble qu'il avait acheté en 1951, au musée d'ethnographie de Rotterdam ${ }^{21}$. C'est probablement à l'occasion d'un voyage à Amsterdam en avril $1961^{22}$, que Brauner se rend pour la première fois dans cette galerie, accompagné de son épouse Jacqueline. Ils établissent tous deux une liste d'objets qu'ils souhaitent acquérir, notamment des «objets totémiques de NouvelleGuinée $^{23}$ ». L'étude des archives de l'artiste et de celles du marchand ${ }^{24}$ montre que sur les vingt-deux pièces océaniennes présentes dans le legs, dix-sept d'entre elles ont été acquises à la galerie Lemaire.

\section{La collection, un répertoire de formes}

6 Au début des années 1960, l'œuvre de Brauner tend vers une simplification et un aplanissement plus prononcés des formes. Cette évolution coïncide avec la période du développement de sa collection et plus particulièrement de l'acquisition d'une typologie d'objet bien précise: les armes cérémonielles d'Afrique centrale. Depuis longtemps collectées par les explorateurs et administrateurs coloniaux, comme trophées ou objets de curiosités, les armes arrivent par caisses entières en Europe dans les années 1950. Elles prennent de la valeur aux yeux des artistes grâce à l'action des marchands qui les hissent au statut de pièces « collectionnables » en les présentant sur des socles et en les polissant. L'intérêt des artistes pour ces objets s'illustre notamment à travers l'exposition L'art de l'Afrique Noire et "l'époque nègre" de quelques artistes contemporains, organisée en 1956 par Maurice Allemand au musée d'Art et d'Industrie de Saint-Étienne. Dans le catalogue de celle-ci, figurant dans la bibliothèque de Brauner, sont reproduites six armes africaines prêtées par les marchands Le Corneur- 
Roudillon et Charles Ratton. Brauner rejoint l'engouement de ses contemporains en acquérant six couteaux d'Afrique centrale.

Fig. 2 et 3
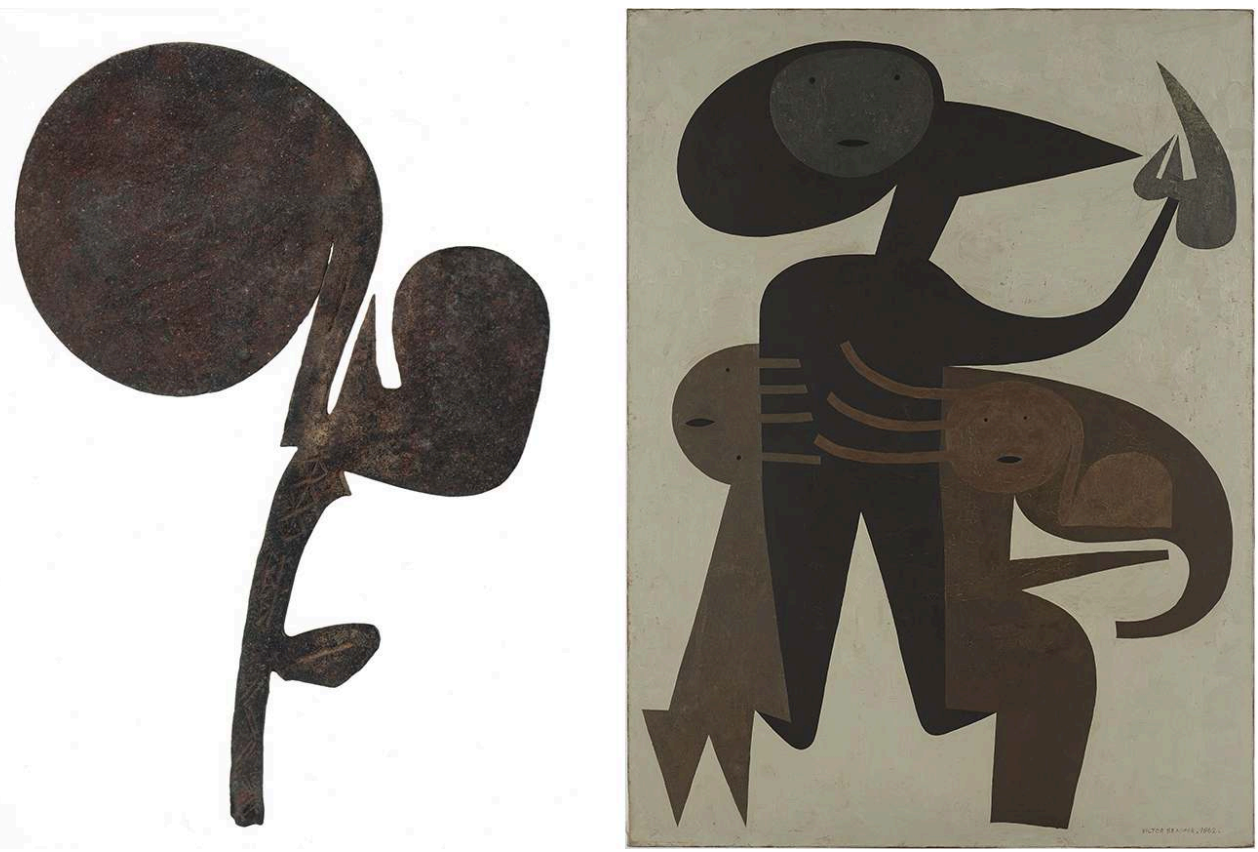

A gauche : Couteau à fonction d'échange, Banda, République centrafricaine, $X X$ e siècle, fer gravé, 26,5 × $19 \times 1 \mathrm{~cm}$, anc. coll. Victor Brauner, Saint-Étienne, MAMC, inv. 90.31.50.

(c) Musée d'art moderne et contemporain de Saint-Étienne Métropole, photo Yves Bresson.

A droite : Victor Brauner, La Formatrice, 1962, huile sur toile, $130 \times 97 \mathrm{~cm}$, Paris, Centre Pompidou, MNAM-CCl, inv. AM 1974-26.

(c) Adagp 2019, Paris. Centre Pompidou, MNAM-CCI / Bertrand Prévost / Dist. RMN-GP 
Fig. 4

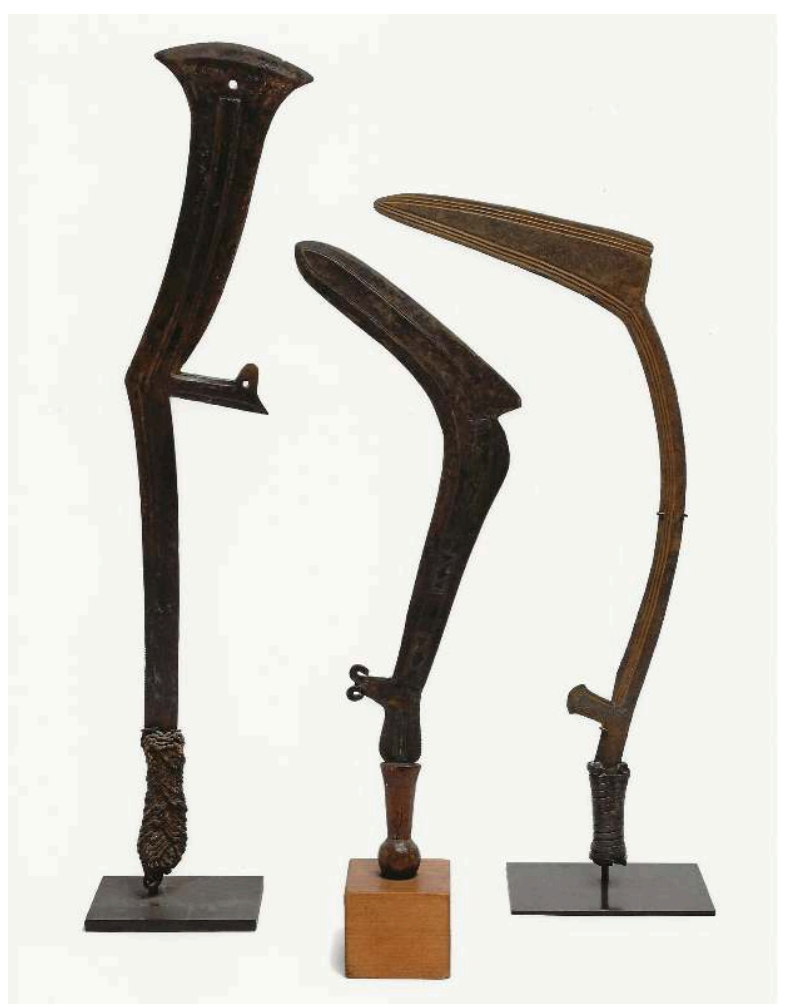

De gauche à droite : couteau de jet za, Cameroun, République centrafricaine, Congo-Brazzaville, fer gravé, cuir ou fibre ; arme faucille magia, Banda, Congo (RDC), République centrafricaine, fer gravé, bois ; arme faucille, Bandia, Binja, Congo (RDC), fer gravé, cuivre, anc. coll. Victor Brauner, coll. Samy Kinge [identifications de l'auteure].

(c) Samy Kinge

Ces armes sont utilisées par l'artiste comme des sources d'inspiration pour renouveler son vocabulaire formel. Une photographie prise dans l'atelier de Brauner ${ }^{25}$, à Varengeville-sur-Mer, le montre bien. Posé sur une table parmi les pinceaux, un couteau en fer gravé façonné par les populations Banda (fig. 2), présentes en République centrafricaine, trône parmi des toiles qui semblent en être directement inspirées, notamment La Formatrice, peinte en 1962 (fig.3). Les trois figures anthropomorphes qui s'y entremêlent reprennent les formes à la fois arrondies et pointues du couteau banda ainsi que la silhouette de l'arme cérémonielle réalisée par les Kota du Gabon ${ }^{26}$, également dans la collection de Brauner. Ces deux pièces, auxquelles s'ajoute un couteau de jet banda, ont été léguées au musée d'art moderne et contemporain de Saint-Étienne. Brauner avait également en sa possession trois autres armes (fig.4), peu connues elles sont actuellement conservées dans la collection personnelle du galeriste Samy Kinge, spécialiste de l'œuvre de l'artiste ${ }^{27}$. Deux d'entre elles sont des faucilles. Par ses gravures caractéristiques et sa poignée se terminant par une boule, l'une est proche des faucilles appelées magia des Banda de la République Démocratique du Congo et de la République centrafricaine. Par l'excroissance située audessus de sa poignée, l'autre faucille est proche de celles des Bandia et des Binja, et de plusieurs autres populations voisines, du centre de la République Démocratique du Congo et des régions limitrophes en République centrafricaine. La troisième arme est un couteau de jet, nommé za, provenant de populations établies dans une vaste région allant du sud-est du Cameroun, en passant par l'est de la République centrafricaine 
jusqu'au nord du Congo-Brazzaville. Moins courbé que les armes précédentes, il se distingue par la forme bombée de sa partie tranchante.

Au cours de l'année 1961, les silhouettes de ces trois objets se retrouvent fréquemment dans les peintures de Brauner. L'étude pour l'œuvre Vases communicants (fig. 5) en est un exemple ${ }^{28}$. Au centre de la fusion de deux corps se détache un amalgame d'où émergent des figures en " positif » et en «négatif ». La silhouette arrondie et pointue $\mathrm{du}$ couteau banda est bien visible au centre, tout comme celle des faucilles, avec leurs lames allongées et courbées se terminant par une excroissance arrondie. Les corps sont affranchis des règles anatomiques et se transforment en signes où les attributs sexuels prédominent. Brauner rend sans doute hommage à l'ouvrage du même titre écrit par Breton en 1932, Les Vases communicants, dans lequel les fantasmes et la sexualité refoulés trouvent leur expression dans les rêves. Contrairement à l'érotisme libéré des règles de convenances de la série Victor Victorel réalisée en 1949, les corps s'érotisent dans une abstraction géométrique où les formes s'interpénètrent et jouent sur des contrastes pour mieux se rendre visibles.

Fig. 5

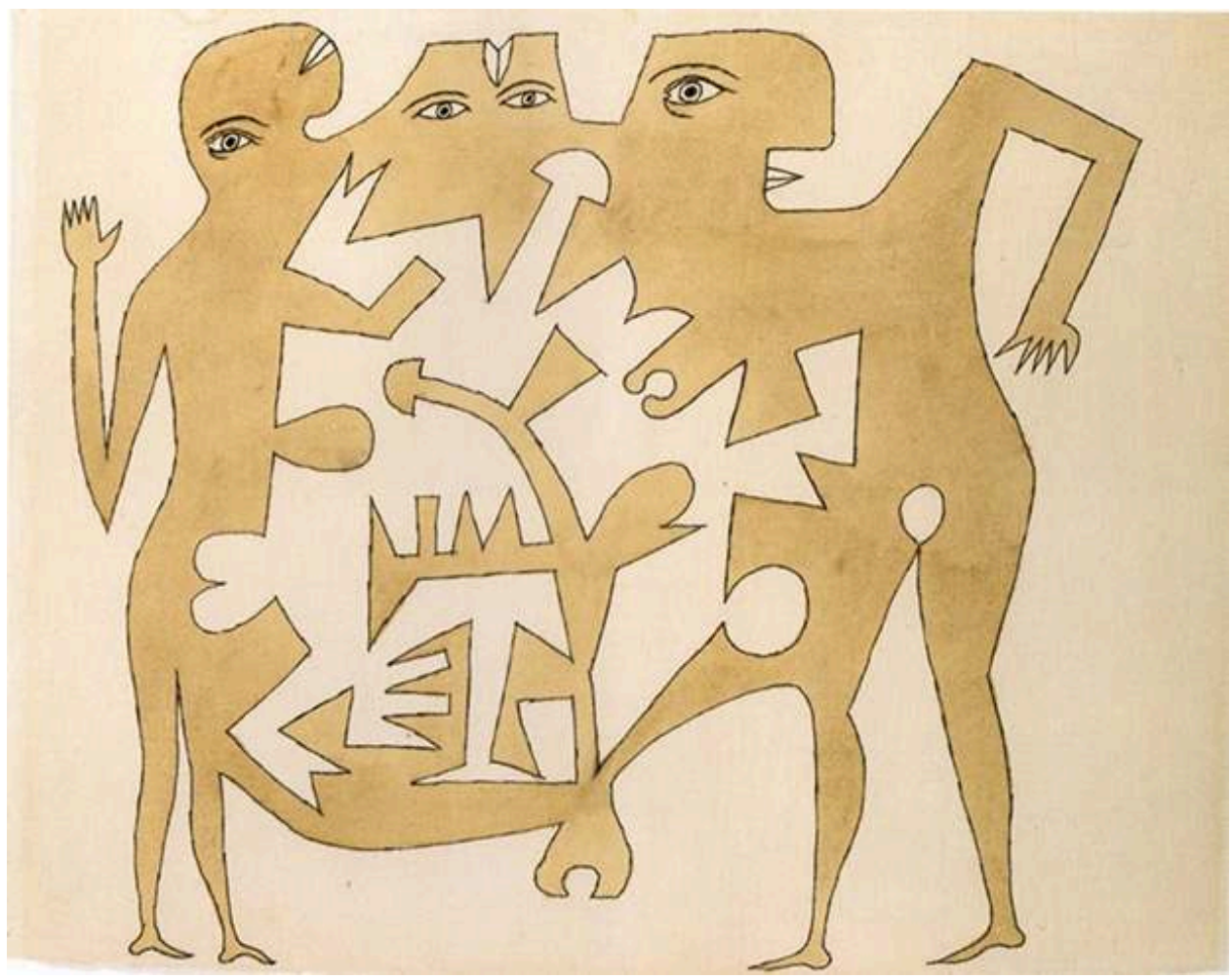

Victor Brauner, Étude pour les Vases communicants, c. 1961, aquarelle et plume, 49,5 × $61 \mathrm{~cm}$, coll. Samy Kinge.

(c) Samy Kinge, photo Jean-Louis Losi

\section{Un regard nourri par des connaissances « ethnographiques »}

Considérer uniquement l'angle formel pour analyser le rapport entretenu par Brauner aux arts extra-européens est réducteur. Le développement de l'ethnographie et de 
l'ethnologie comme sciences, avec la fondation de l'Institut d'ethnologie par Marcel Mauss, Paul Rivet et Lucien Lévy-Bruhl en 1925, et les premières collectes scientifiques qui en découlent ont favorisé une meilleure connaissance des conditions de création et des significations des objets rapportés en Occident. Ce phénomène a eu un impact sur la réception des pièces par les artistes et particulièrement sur le mouvement surréaliste émergeant dans les mêmes années ${ }^{29}$. L'étude des sociétés alors qualifiées de " primitives » rend compte d'autres modes de fonctionnement qui remettent en cause les valeurs de la civilisation occidentale. Les surréalistes s'appuient notamment sur La Mentalité primitive de Lévy-Bruhl, publié en 1922, pour légitimer leurs propres intuitions autour du rêve et de l'inconscient, des lieux où s'exprimerait l'imagination sous ses formes les plus primordiales. En 1935, l'anthropologue écrit La mythologie primitive. Le monde mythique des australiens et des papous dont Brauner possède un exemplaire. Durant les années 1941 et 1942, l'artiste y fait référence à de nombreuses reprises. Deux éléments retiennent son attention: les coiffures dori et gari ${ }^{30}$. Brauner est particulièrement intéressé par la dimension transculturelle que confère Lévy-Bruhl à ces objets. Selon ce dernier, des motifs semblables à ces coiffures, portées lors de rituels de fécondité en Nouvelle-Guinée, se retrouvent sur les peintures rupestres du nordouest de l'Australie et possèdent une même signification. Brauner s'approprie cette symbolique en réinterprétant la coiffe sous la forme d'un masque-heaume qu'il intègre dans un projet de spectacle, lors duquel l'objet est porté par une femme dans une scène nommée la « danse de l'appropriation du fleuve ${ }^{31}$ ».

Les études d'ethnologie lues par Brauner nourrissent son imaginaire, mais lui serventelles à documenter sa collection? L'acquisition d'œuvres est concomitante à celle de livres. L'état dans lequel nous est parvenue sa bibliothèque, bien que partiellement représentatif de ses lectures et ne prouvant pas qu'il ait consulté la totalité des ouvrages, ni approuvé leur contenu, donne accès à l'univers intellectuel dans lequel il évolue. Brauner possède de nombreux livres consacrés aux arts et coutumes des populations d'Amérique du Nord et du Sud, d'Afrique, d'Océanie, d'Égypte, et d'Asie. L'étude de Marcel Griaule, intitulée Dieu d'eau : entretiens avec Ogotemmêli, parue en 1948, est-elle une référence pour l'aider à comprendre la signification des huit pièces dogon qu'il possède ? Difficile à dire. Sa curiosité à l'égard des œuvres qu'il collectionne se révèle de manière explicite à travers les renseignements qu'il inscrit au dos des photographies des sculptures océaniennes que lui envoie le marchand Lemaire. Sur les versos des dix-huit photographies figurent des étiquettes apposées par le galeriste, et sur certaines d'entre elles, des inscriptions écrites par Brauner. Le marchand fournit le contexte culturel dans lequel l'objet a été créé avant son importation en Europe et l'artiste y ajoute ses propres notes. À plusieurs reprises, Brauner complète les informations. C'est notamment le cas pour le masque mei du Moyen Sépik, où il ajoute l'usage, "Masque d'initiation », et l'ancienneté, «+60 ans ». D'où proviennent ces données supplémentaires? Se renseigne-t-il dans des ouvrages ou auprès d'amis collectionneurs? Ou encore auprès de la galerie elle-même? Les annotations de Brauner révèlent un autre aspect de son appréhension des arts extra-européens. Contrairement à ce qu'il avance lors d'un entretien en 1965, sur lequel nous reviendrons, le plaisir esthétique n'est pas l'unique critère qui guide ses achats. Ses choix s'affinent par la connaissance de l'usage in situ des pièces, de leur ancienneté et de leur provenance. Les acquisitions de Brauner ne résultent donc pas d'une rencontre hasardeuse mais d'une minutieuse préparation. Un processus qui correspond aux 
transactions effectuées avec la galerie Lemaire mais qui semble être différent pour ses achats parisiens qui n'ont laissé aucune trace dans ses archives.

\section{Magie et animisme}

11 Dans leur enquête sur les collectionneurs d'arts extra-européens, Brigitte Derlon et Monique Jeudy-Ballini ont montré que, pour la plupart d'entre eux, l'objet possède un "pouvoir émotionnel », il est "une présence, une entité souvent créditée de formes d'action ou d'intentionnalitée ${ }^{22} »$. Lorsqu'il est interrogé sur sa collection, Brauner ne tient pas ce discours. Il a cependant des propos similaires lorsqu'il parle de ses propres œuvres. Dans un entretien accordé à Alain Jouffroy, il confie que ses tableaux possèdent «un sens d'envoûtement, de contre-envoûtement, de magie, d'attirance ou de protection $^{33}$ ». Sensible à la tradition kabbalistique et aux sciences occultes, Brauner attribue aux matériaux des propriétés spécifiques qui confèrent à ses œuvres la faculté d'agir sur le réel, de manière positive ou négative. En témoignent les objets conjuratoires qu'il fabrique pendant la Seconde Guerre mondiale, comme ces petites boîtes dans lesquelles il dispose divers matériaux (de la terre crue, du fil de fer, de la cire) et des symboles ésotériques correspondant à l'accomplissement de formules précises $^{34}$. La matière possède également une forme de primitivité. Selon Brauner, elle est «la clé des grandes nostalgies et des grandes rêveries qui font la création ${ }^{35}$ » et permet $\mathrm{d}^{\prime}$ " arriver à la source des choses $^{36}$ ». Le tableau Origines de l'imagination de la matière ${ }^{37}$, réalisé à la cire en 1958 , illustre cette conception. Par l'usage de ce matériau lié au règne végétal et animal, récurrent dans sa pratique, Brauner rejoint les Universaux de la création artistique. La pensée braunérienne est proche de celle de Gaston Bachelard ${ }^{38}$ et de ses essais sur "l'imagination matérielle " dans lesquels il développe une théorie autour de l'intentionnalité de la matière, les quatre éléments naturels étant considérés comme les sources de l'inspiration poétique ${ }^{39}$.

En 1941, Brauner écrit une fiction, intitulée Promenade ${ }^{40}$, dont l'analyse offre des clés de lecture pour appréhender son rapport aux objets. Le récit narre l'aventure nocturne d'un peintre qui a emporté avec lui une collection de vingt-cinq objets, issus du quotidien et de la nature. La vision de tous ces objets provoque chez le narrateur un désir urgent : «Je me précipitai [pour] prendre la collection qui se trouvait devant moi et qu'à tout prix je devais posséder ${ }^{41}$. ». Il précise ensuite : «je me trouvai exalté et l'on [aurait] dit que le contact de ces objets amplifi[ait] ma force ${ }^{42}$.». Ce texte réunit les caractéristiques propres aux collectionneurs d'arts extra-européens, à savoir : le désir de possession, les fortes émotions suscitées par la vision des objets, et la capacité agissante qui leur est attribuée ${ }^{43}$. Après-guerre, la collection d'arts extra-européens se substitue aux talismans protecteurs. Brauner confère-t-il aux œuvres d'Afrique subsaharienne, d'Océanie et d'Amérique du Sud la même intentionnalité qu'il attribue à ses propres œuvres et aux objets qui l'entourent? Croyant aux transferts d'âmes et d'esprits dans des objets inanimés, des plantes ou des animaux, Brauner a une conception animiste du monde. Un de ses écrits daté de 1962 est éclairant à ce sujet : "OBJET, tu me donnes de ta survivance je te donne de mon âme et ainsi nous vaincrons la mort ${ }^{44} . . . »$. Sigmund Freud, dans Totem et tabou : interprétation par la psychanalyse de la vie sociale des peuples primitifs, publié en 1913 et traduit en français en 1924, décrit le système animiste comme une réponse à un besoin pratique de soumettre le monde à la volonté de l'homme. Celui-ci y parvient en ayant recours à la magie et à la sorcellerie, 
deux techniques qui lui permettent de contrôler les phénomènes de la nature et d'agir contre ce qui lui est néfaste. Pour décrire les différents types de magie, Freud s'appuie sur l'étude de James George Frazer intitulée Le Cycle du Rameau d'or : le roi magicien dans la société primitive ${ }^{45}$, un ouvrage fondateur de l'anthropologie en Europe présent dans la bibliothèque de Brauner et auquel il se réfère pour confectionner ses amulettes protectrices pendant la guerre. Ce regard "magique» et la part animiste de sa conception du monde participent à définir sa relation à sa collection d'arts extraeuropéens.

\section{Un « primitivisme sans emprunts ${ }^{46}$ " et une relation de fraternité avec les arts extra-européens}

En 1965, interrogé sur le rapport qu'il entretient avec les œuvres extra-européennes de sa collection Brauner répond ceci :

« Il y a évidemment une fraternité entre ces objets et nous-même. Et ce ne sont pas si vous voulez des références directes mais ce sont des références de parentés, qui me servent évidemment parce que ce sont d'abord des objets admirables à mon sens ne serait-ce que sur le plan esthétique [...] Mais je me trouve moi familier à ces objets sans que ces objets, comment est-ce que je peux dire, m'ait influencé d'une manière directe ${ }^{47}$. »

Ce discours réunit les deux types de relation que les artistes occidentaux ont pu avoir avec les arts extra-européens : une relation formelle, mis en exergue par l'exposition de William Rubin en 1984 au Museum of Modern Art de New York, "Primitivism" in 20th Century Art. Affinity of the Tribal and the Modern, et une relation qui serait à saisir sur le plan psychologique, défendue par Robert Goldwater en 1966, le premier historien de l'art à avoir étudié la question du primitivisme. En effet, Brauner énonce que les objets lui «servent » sur le plan esthétique et précise ensuite qu'il n'y a pas eu d'influence directe, mais qu'il existe une familiarité et une fraternité dans les démarches créatrices. Les propos de Brauner témoignent d'un type de relation que l'anthropologue Carlo Severi a défini sous l'appellation « primitivisme sans emprunts ${ }^{48}$ ». C'est-à-dire un primitivisme qui serait fondé sur les actes qui mènent à l'œuvre et sur l'identification de l'artiste avec une vision utopique du monde tournée vers le passé, et non plus sur les formes qui la constituent.

La dimension fraternelle exprimée par Brauner va de pair avec sa croyance en l'existence d'un fonds commun à l'humanité. Dans un entretien avec Edouard Roditi en 1963, l'artiste confie adhérer aux théories de Carl Gustav Jung concernant l'existence d'un inconscient collectif universel qui s'exprimerait sous la forme d'archétype ${ }^{49}$. Selon la pensée jungienne, l'archétype est un symbole primitif et universel qui se manifeste notamment dans les mythes. Il existerait ainsi des figures communes à toutes les cultures. Une conception que partage Brauner lorsqu'il affirme qu'il n'y a "pas de création de formes nouvelles ${ }^{50}$ » mais des «formes archaïques permanentes ${ }^{51}$ ». Il remarque également que les artistes contemporains cherchent à exprimer ce répertoire visuel en créant "une continuité entre les valeurs les plus anciennes des manifestations de l'art de l'homme et celles d'aujourd'hui ${ }^{52}$ » et désigne cette tendance par le terme " néoarchaïsme ${ }^{53}$ ». Les objets qu'il qualifie de " primitifs » sont placés au cœur de ce processus car ce sont eux «qui permettent de rejoindre les civilisations passées $^{54}$ ». L'art acquiert alors une dimension holistique dépassant les frontières 
temporelles et géographiques établies par l'histoire de l'art occidental traditionnelle. Le paradigme primitiviste, qui recherche des emprunts formels et des analogies, n'opère plus. En affirmant qu'il n'y a pas de formes inférieures à d'autres, Brauner place sur un pied d'égalité toutes les productions artistiques. Néanmoins, son discours est empreint d'un racisme culturel toujours prégnant à l'époque. En recherchant dans l'Autre une dimension originelle et élémentaire, incarnée par les qualités de pureté, d'authenticité et de liberté créatrice, il projette sur les artistes non occidentaux son propre fantasme. Il voit en eux les qualités qu'il souhaite posséder et ne prend pas en compte les réalités conceptuelles qui ont présidé à la création des œuvres, régies selon des règles et des codes précis.

Brauner côtoie des artistes et des intellectuels qui tentent de repenser les modalités d'un dialogue entre les cultures. Dans les années 1955, il fréquente notamment la galerie du Dragon où exposent des artistes de nationalités diverses, citons entre autres le chilien Roberto Matta, le cubain Agustin Cárdenas, et le mexicain Enrique Zañartu. C'est sans doute dans ce lieu d'émulation cosmopolite qu'il rencontre Édouard Glissant avec lequel il entretient une forte amitié. La réflexion du poète martiniquais autour d'une identité définie dans sa relation à l'autre, et non plus uniquement dans son unicité, paraît avoir imprégné l'artiste roumain. Comme de nombreuses personnalités qui l'entourent, Brauner s'engage dans la lutte pour la décolonisation en soutenant l'indépendance des Algériens. Il participe à l'« Anti-procès pour le droit de l'homme à disposer de lui-même ", organisé par Alain Jouffroy et Jean-Jacques Lebel en 1960, à la galerie des Quatre Saisons, à Paris ${ }^{55}$. Brauner a une double posture face aux arts extraeuropéens, collectionnant des œuvres à une époque où elles affluent en Europe dans des conditions plus ou moins légales et prenant position en faveur de l'indépendance des peuples colonisés ${ }^{56}$.

La relation entretenue par Brauner à sa collection d'arts extra-européens est de nature plurielle. Regard formel, ethnographique, «magique» et «fraternel » se rejoignent. Mis à part quelques emprunts formels directs, en particulier les armes d'Afrique de l'Ouest, l'agentivité des œuvres collectionnées sur la création de Brauner a surtout opéré sur le plan « de l'accompagnement et de l'encouragement ${ }^{57}$ ». Quelque mois avant son décès, Brauner confie à Pierre Dumayet : «ce sont les civilisations qui m'ont plagié, alors je les ai acceptées très fraternellement dans ma collaboration ${ }^{58} »$. Ce plagiat par anticipation, exprimé avec humour, signifie pour l'artiste qu'il ne s'agit pas de s'interroger en termes d'influence, mais que des formes similaires sont susceptibles d'émerger lorsqu'un même état d'esprit préside à leur élaboration ${ }^{59}$. Les œuvres d'Afrique subsaharienne, d'Océanie et d'Amérique du Sud que Brauner s'approprie sont des supports matériels qui lui permettent de rejoindre un temps mythique où s'exprimerait la permanence des formes entre les civilisations, et par là atteindre la dimension universelle de la création. Par sa collection, son œuvre et son discours, Brauner est un des acteurs de l'histoire de la relation des artistes occidentaux aux arts extra-européens qui se poursuit après la Seconde Guerre mondiale, une période qui reste à étudier pour en comprendre les spécificités. 


\section{NOTES}

1. Figure de masque karanga, Mossi, Burkina Faso, Afrique, $\mathrm{xx}^{\mathrm{e}}$ siècle, bois polychrome brun-rouge et blanc et corde, $87 \times 78 \times 23 \mathrm{~cm}$, anc. coll. Brauner, Saint-Étienne, MAMC, inv. 90.31.22.

2. Crochet double de suspension, Iatmul, Moyen Sépik, Papouasie-Nouvelle-Guinée, Océanie, $\mathrm{xx}^{\mathrm{e}}$ siècle, bois dur patiné, $49 \times 17 \times 4 \mathrm{~cm}$, anc. coll. Brauner, Saint-Étienne, MAMC, inv. 90.31.2.

3. Fragment de sculpture à visage anthropomorphe, Vanuatu, Océanie, $\mathrm{XIX}^{\mathrm{e}}-\mathrm{xx} \mathrm{x}^{\mathrm{e}}$ siècle, bois délavé, $82 \times 11,5 \times 6 \mathrm{~cm}$, anc. coll. Brauner, Saint-Étienne, MAMC, inv. 90.31.12.

4. Victor Brauner, Torchon peint, 1964, peinture sur tissu, $70 \times 52 \mathrm{~cm}$, Strasbourg, musée d'art moderne et contemporain, inv. 55.988.1.30 (1).

5. «Quelle est l'influence des arts primitifs sur la peinture d'aujourd'hui? », Arts spectacles, $\mathrm{n}^{\circ} 608,1957, \mathrm{~N}$. p.

6. Henri Meschonnic, Modernité Modernité, Paris, Verdier, 1988, pp. 273-284.

7. Marina Vanci-Perahim, "Ancêtres nécessaires et "douaniers" sans frontières: internationalisation du modèle primitif », dans Gérard Monnier, José Vovelle (dir.), Un art sans frontières. L'internationalisation des arts en Europe (1900-1950), Paris, Publications de la Sorbonne, 1994, pp. 47-63.

8. Cat. d'exp., Dada Africa. Sources et influences extra-occidentales, sous la direction de Ralf Burmeister, Cécile Debray, Michaela Oberhofer, Paris, Musée de l'Orangerie, 18 oct. 2017-19 fév. 2018, Paris, Hazan/Musée de l'Orangerie, 2017.

9. Brauner est écarté du mouvement surréaliste en 1948, suite à son refus de signer l'exclusion du peintre chilien Roberto Matta, puis réintègre le groupe en 1959.

10. La définition de la notion de primitivisme est plurielle et possède une définition élargie qui englobe les productions artistiques de ceux qui sont antérieurs à la civilisation occidentale et ceux qui lui sont extérieurs (les peintres « naïfs ", les enfants, les productions du milieu asilaire).

11. Daniel Sherman, Le primitivisme en France et les fins d'empire (1945-1975), Dijon, Les presses du réel, 2018.

12. Benoît de L'Estoile, Le Goût des Autres. De l'Exposition coloniale aux Arts premiers, Paris, Flammarion, 2007.

13. Jean Laude, La Peinture française (1905-1914) et "l'art nègre ». Contribution à l'étude des sources du fauvisme et du cubisme, $2^{\mathrm{e}}$ éd. rev. et augm., Paris, Klincksiek, 2006 ; Philippe Dagen, Le peintre, le poète, le sauvage. Les voies du primitivisme dans l'art français, $2^{\mathrm{e}}$ éd., Paris, Flammarion, 2010.

14. William Rubin (dir.), Le primitivisme dans l'art du $\mathrm{xx}^{\mathrm{e}}$ siècle. Les artistes modernes devant l'art tribal, éd. française Jean-Louis Paudrat (dir.), Paris, Flammarion, 1991 : la France est peu représentée dans le chapitre consacré à l'art des années 1950-1970.

15. Evan Maurer, « Dada et surréalisme », dans W. Rubin, ibid., pp. 576-577.

16. Jacques Beauffet, "Victor Brauner et les Arts Premiers », dans cat. d'exp., Victor Brauner, un surréaliste européen, sous la direction de Chantal Fernex de Mongex, Chambéry, Musée des Beaux-Arts, 12 oct. 2007-14 janv. 2008, Clermont-Ferrand, éd. Un, 
deux, quatre, 2007, pp. 68-71 ; cat. d'exp., Dialogue des mondes. Victor Brauner et les arts primitifs, sous la direction de Christophe de Fabry, Paris, Galeries Schoffel-Valluet \& Samy Kinge, 21 oct.-4 déc. 2010, Paris, éd. Galerie Schoffel-Valluet/Galerie Samy Kinge, 2010.

17. Leur amitié est perceptible dans les messages qu'elle a inscrit dans ses recueils de poésie offerts à Brauner (Rapaces, 1960, et Le carré blanc, 1965, Fonds Brauner, Paris, Bibliothèque Kandinsky).

18. Une seule lettre de Loeb à Brauner figure dans les archives Brauner conservées à la Bibliothèque Kandinsky, Paris (voir Camille Morando et Sylvie Patry (dir.), Victor Brauner : écrits et correspondances 1938-1948. Les archives de Victor Brauner au Musée national d'art moderne, Paris, Centre Pompidou/INHA, 2005, p. 320). Les archives de la galerie Pierre Loeb sont en cours de dépôt à la Bibliothèque Littéraire Jacques Doucet, Paris.

19. Explosion du silence V, novembre 1956, huile sur toile, coll. part., et Sans titre, 1960, huile et aquarelle sur panneau, coll. part.

20. Entretien de l'auteure avec Jean Roudillon, 31 mai 2018, Paris.

21. Raymond Corbey, Tribal Art Traffic. A Chronics of Taste, Trade and Desire in Colonial and Post-Colonial Times, Amsterdam, Royal Tropical Institute, 2000, pp. 43-45.

22. Voyage mentionné sur un croquis à la plume et à l'encre de Chine, Fonds de dessins Brauner, Cabinet d'arts graphiques, Saint-Étienne, MAMC, inv. 90-10-(5).

23. Lettre de Jacqueline Brauner à Tom Bouman, Varengeville-sur-Mer, 27 février 1962. Archives Brauner, Saint-Étienne, MAMC.

24. Nous remercions Finette Lemaire, petite-fille de Matthias Louis Lemaire.

25. Anonyme, L'atelier de Victor Brauner à Varengeville, [ap. 1962], Fonds Brauner, Album biographique, Dos. Atelier, n 3.83 TO, Paris, Bibliothèque Kandinsky.

26. Arme cérémonielle, Kota, Gabon, Afrique, $\mathrm{xx}^{\mathrm{e}}$ siècle, fer gravé, manche en bois entouré de fil de laiton, $50 \times 36 \times 4 \mathrm{~cm}$, anc. coll. Brauner, Saint-Étienne, MAMC, inv. 90.31.54.

27. Nous remercions Samy Kinge pour la découverte de ces pièces. Pour identifier ces trois armes nous nous sommes appuyés sur l'ouvrage de Jean Elsen, De fer et de fierté. Armes blanches d'Afrique noire du musée Barbier-Mueller, Genève/Milan, Musée BarbierMueller/Cinq Continents, 2003.

28. L'œuvre Vases communicants (1961, huile sur toile, $89,2 \times 116,3 \mathrm{~cm}$ ) est actuellement conservée en collection privée. Elle est reproduite dans le cat. d'exp., Victor Brauner, Paris, Galerie Rive Droite, 1962, N. p.

29. James Clifford, Malaise de la culture. L'ethnographie, la littérature et l'art au Xxe siècle, Paris, École nationale supérieure des beaux-arts, 1996, chap. 4 «Du surréalisme ethnographique ", pp. 121-152.

30. Au sujet de la récurrence de ces motifs, voir C. Morando et S. Patry, op. cit. note 18, p. 75 , note 33 .

31. Victor Brauner, Sans titre, 1942, reproduction photographique d'un dessin à la plume et encre violette sur papier à lettres, Fonds Brauner, Albums dessins, BRAU 39, Paris, Bibliothèque Kandinsky. Dessin original conservé au MAMC, Saint-Étienne, inv. 90.10.1423.

32. Brigitte Derlon et Monique Jeudy-Ballini, La passion de l'art primitif: enquête sur les collectionneurs, Paris, Gallimard, 2008, p. 156. 
33. Victor Brauner, entretien avec Alain Jouffroy, «Victor Brauner peint un tableau devant vous ", Connaissance des Arts, $n^{\circ}$ 107, janvier 1961, p. 88.

34. Camille Morando, «Matérialités magiques des objets à la finalité talismanique chez deux artistes autour du surréalisme en regard de l'art brut » dans Jill Carrick, Fabrice Flahutez, Pauline Goutain (dir.), Dimensions de l'art brut. Une histoire des matérialités, Paris, Presses universitaires de Paris Nanterre, 2017, pp. 49-57

35. Transcription de Luce Hoctin d'un manuscrit de Brauner, 1958, Fonds Brauner, Ms 47 inv. 8818.1115.5, Paris, Bibliothèque Kandinsky.

36. Id., Ibid.

37. Victor Brauner, Origines de l'imagination de la matière, cire, 1958, coll. non identifiée, Fonds Brauner, Album d'œuvres, BRAU 61, n 11, Paris, Bibliothèque Kandinsky.

38. Brauner a dans sa bibliothèque un livre de Bachelard: La poétique de l'espace, Paris, PUF, 1958.

39. Barbara Puthomme, Le Rien profond. Pour une lecture bachelardienne de l'art contemporain, Paris, L'Harmattan, 2002.

40. Victor Brauner, Promenade, 28 juin-26 oct. 1941, publié dans C. Morando et S. Patry, op. cit. note 18, pp. 49-55.

41. Id., Ibid., p. 51.

42. Id., Ibid.

43. B. Derlon et M. Jeudy-Ballini, op. cit. note 32.

44. Victor Brauner, Codex d'un visage, 1962, publié dans cat. d'exp., Victor Brauner, Paris, Galerie Didier Imbert Fine Art, 26 oct.-21 déc. 1990, Paris, éd. Didier Imbert Fine Art, 1990, p. 28.

45. Le Cycle du Rameau d'or de James George Frazer paraît en 1890. Brauner a dans sa bibliothèque l'édition en deux volumes de 1935 (Paris, P. Geuthner).

46. Carlo Severi, "L'empathie primitiviste», Images Re-vues, hors-série 1, 2008. Consultable : www.journals.openedition.org/imagesrevues/794 [28/02/2018].

47. Victor Brauner, entretien avec Adam Saulnier, émission de télévision Les expositions, [enregistrement vidéo], diffusé le 31 janvier 1965, Archives de l'INA.

48. C. Severi, art. cité note 47 .

49. Edouard Roditi, « Entretien avec Victor Brauner (1963)», Propos sur l'art, Paris, José Corti, 1987, p. 14. Brauner possède cinq ouvrages de Jung dans sa bibliothèque.

50. Victor Brauner, entretien avec Françoise Dumayet, émission de télévision Terre des Arts [retranscription], 1961, Fonds Brauner, inv. 8818.1125, Paris, Bibliothèque Kandinsky.

51. V. Brauner, entretien avec A. Jouffroy, op. cit. note 33, p. 88.

52. Victor Brauner, Projet de lettre, destinataire inconnu, Golfe-Juan, juillet 1953, Fonds Brauner, inv. 8818.1086.1, Paris, Bibliothèque Kandinsky.

53. Id., Ibid.

54. V. Brauner, entretien avec A. Jouffroy, op. cit. note 34, p. 88.

55. Tract pour l'Anti-procès, Fonds Brauner, inv. 8818.718, Paris, Bibliothèque Kandinsky. 
56. Sophie Leclercq, La rançon du colonialisme. Les surréalistes face aux mythes de la France coloniale, 1919-1962, Dijon, Les presses du réel, 2010.

57. P. Dagen, op. cit. note 13, p. 546.

58. Victor Brauner, entretien avec Pierre Dumayet, émission de télévision Lectures pour tous, [enregistrement vidéo], diffusée le 26 janvier 1966, Archives de l'INA.

59. Didier Semin, «Le rêve d'un alchimiste en Océanie: Victor Brauner et la pensée sauvage », dans cat. d'exp., Dialogue des mondes, op. cit. note 17, pp. 17-23.

\section{RÉSUMÉS}

Constituée pour une grande partie entre le milieu des années 1950 et le début des années 1960, la collection de l'artiste Victor Brauner (1903-1966) est principalement composée d'œuvres originaires d'Afrique de l'Ouest et de Papouasie-Nouvelle-Guinée. Actuellement conservée au musée d'art moderne et contemporain de Saint-Étienne Métropole, la collection a peu été étudiée, tout comme la relation de l'artiste à celle-ci. Cet article se propose de mettre en lumière la constitution de la collection par Brauner. L'artiste côtoie la seconde génération de marchands parisiens qui émerge au lendemain de la Seconde Guerre mondiale et acquiert également des œuvres à Amsterdam. Il s'agira ensuite d'analyser les différents types de regards, à la fois distincts et complémentaires, qu'il a portés sur les œuvres collectionnées - d'ordre formel, intellectuel, «magique » et «fraternel » - afin de rendre compte de la nature plurielle de ses rapports aux arts extra-européens et du dialogue qui s'instaure entre sa création et ceux-ci.

Put together mostly between the mid-1950s and the early 1960s, the collection of the artist Victor Brauner (1903-1966) is mainly made up of works from West Africa and Papua New Guinea. Now in the Musée d'Art Moderne et Contemporain de Saint-Étienne Métropole, the collection has been studied little as has the artist's relationship to it. This article aims to shed light on Brauner's putting together of the collection. The artist frequented the second generation of Parisian markets that emerged after the Second World War and also acquired works in Amsterdam. There will also be an analysis of the various ways, both distinct and complementary, he regarded the works collected -formally, intellectually, "magically" and "fraternally" - to give an account of the plural nature of his relationship to extra-European art and of the dialogue between it and his own work.

\section{INDEX}

Keywords : Victor Brauner, artist-collector, West Africa, Papua New Guinea, art market, extraEuropean, primitivism, surrealism

Mots-clés : Victor Brauner, artiste collectionneur, Afrique de l'Ouest, Papouasie-NouvelleGuinée, marché de l'art, primitivisme, surréalisme 


\section{AUTEUR}

\section{NOÉMIE FILLON}

Diplômée du deuxième cycle de l'École du Louvre, parcours « recherche » en histoire de l'art appliquée aux collections, Noémie Fillon a intégré ce cursus en troisième année du premier cycle pour étudier la spécialité histoire des arts de l'Afrique subsaharienne. Elle a soutenu en 2018 un mémoire de recherche, sous la direction de Camille Morando, consacré à l'étude de l'œuvre de Victor Brauner à l'aune de sa collection d'arts extra-européens.

A graduate of the École du Louvre (research: art history applied to collections), Noémie Fillon joined this programme to study the history of sub-Saharan African art. In 2018 she a defended a research thesis, supervised by Camille Morando, on the study of the work of Victor Brauner in the light of his collection of extra-European art. 